\title{
Renal Replacement Therapy in Qatar-Past, Present and Future
}

\author{
Hassan Al Malki, Awad Hamed Rashed, Muhammad Asim* \\ Nephrology Division, Department of Medicine, Hamad General Hospital, Hamad Medical Corporation, Doha, \\ Qatar \\ Email: *masim@hamad.qa
}

How to cite this paper: Al Malki, H., Rashed, A.H. and Asim, M. (2018) Renal Replacement Therapy in Qatar-Past, Present and Future. Open Journal of Nephrology, 8, 42-55.

https://doi.org/10.4236/ojneph.2018.82006

Received: April 27, 2018

Accepted: June 11, 2018

Published: June 14, 2018

Copyright (c) 2018 by authors and Scientific Research Publishing Inc. This work is licensed under the Creative Commons Attribution International License (CC BY 4.0).

http://creativecommons.org/licenses/by/4.0/

\begin{abstract}
The economic development of Qatar alongside the resultant lifestyle changes in the last few decades has contributed to increasing rates of obesity, diabetes mellitus and hypertension with consequent increased incidence and prevalence of chronic kidney disease and end-stage-renal-disease (ESRD). This article describes renal replacement therapy (RRT) services in Qatar and their evolution in response to challenges posed by the growth of ESRD with reference to regional and international data. It covers the history of RRT, highlighting significant advances in chronological order, as well as providing an overview of the current status of RRT in the multicultural and socioeconomically diverse society that inhabits Qatar. Finally, it casts a glance into the future, predicting how RRT services will further evolve to address the current limitations.
\end{abstract}

\section{Keywords}

Chronic Kidney Disease, Dialysis, End-Stage Renal Disease, Renal Replacement Therapy, Transplantation, Qatar

\section{Introduction}

Qatar is a peninsula extending north from Saudi Arabia into the Arabian Gulf, covering an overall area of $11,627 \mathrm{~km}$, with a population of 2.5 million [1]. Doha is the capital and most populous city of the State of Qatar. Blessed with the world's third largest natural gas field, Qatar has the world's highest per capita GDP [2]. The economic boom experienced by Qatar in recent years has been associated with a surge in international migration of workers from developing countries, evidenced by an annual population growth of $11.8 \%$ in 2004 and $18.9 \%$ in 2008 . Consequently, approximately $85 \%$ of the population is non-citizen. 
Inappropriate dietary and lifestyle patterns that have accompanied economic development have led to increasing rates of obesity and chronic non-communicable diseases (NCDs). Chronic kidney disease (CKD) and end-stage-renal-disease (ESRD) are key determinants of poor health outcomes of these conditions and put a significant economic burden on health systems.

This article describes the evolution of renal replacement therapy (RRT) services in Qatar, a multi-cultural society with substantial socioeconomic diversity, to combat the challenges posed by the growth of ESRD into an international perspective using information from different renal registries. International comparisons should however be interpreted with caution as data collection methods vary across countries. The incidence and prevalence of RRT in Qatar was analyzed using aggregated data from each hospital/dialysis center; hence adjustment on age, gender, co-morbidities or cause of ESRD was not possible. It is also important to appreciate that prevalence of RRT modalities in a country depends not only on underlying burden of ESRD, but also on access to treatment, healthcare infrastructure, treatment outcomes, and in case of kidney transplantation-organ availability, and cultural beliefs.

\section{Evolution of RRT Services in Qatar}

The evolutionary history of the development of RRTs in Qatar is summarized in Table 1. Although the first hospital of Qatar, Rumailah Hospital, was opened in 1957, dialysis services were not available until 1976; Qatari citizens used to travel and live abroad for maintenance hemodialysis (HD). In 1976, peritoneal dialysis (PD) was started for acute kidney injury (AKI). PD catheters used to be inserted in the procedure room of a medical ward by a medical registrar who had previous international experience in dialysis therapies. Five years later, a 5-station HD unit was established to treat patients with severe AKI or ESRD-this unit was managed by one physician, 2 dialysis nurses and 2 dialysis technicians.

In 1979, Hamad Medical Corporation (HMC) was established. This was responsible for building a network of hospitals and primary health centers that manage the bulk of healthcare in Qatar. Nephrology emerged as a distinctive subspecialty in 1982, at the launch of Hamad General Hospital (HGH). This paved the way for development of inpatient and outpatient nephrology services. A new 24-station HD unit was opened in HGH in 1984 to accommodate the increasing number of ESRD patients, decrease dependence on dialysis abroad and to provide HD support to a growing number of critically ill patients in newly established intensive care units. Continuous ambulatory peritoneal dialysis (CAPD) program was established in $\mathrm{HGH}$ in 1997, offering the patients a home-based therapy with lifestyle benefits. In 2008, a low renal clearance clinic was set up, designed for patients at CKD stages 4 - 5 to carefully plan their RRT. Fahad Bin Jassim Unit-the largest dialysis unit of the country was inaugurated in 2010 in Doha. It has $80 \mathrm{HD}$ stations as well as dedicated areas for training and follow-up of patients on CAPD and automated peritoneal dialysis (APD). 
Table 1. The evolution of renal replacement therapy (RRT) services in Qatar. PD, peritoneal dialysis; $\mathrm{HD}$; hemodialysis, CAPD: continuous ambulatory peritoneal dialysis.

\begin{tabular}{|c|c|}
\hline Year & The evolution of RRT services in Qatar \\
\hline 1976 & First acute PD in Rumailah Hospital \\
\hline 1981 & First HD unit in Rumailah Hospital \\
\hline 1982 & Establishment of nephrology subspecialty in Hamad Medical Corporation \\
\hline 1984 & Launch of first dialysis unit in Hamad General Hospital \\
\hline 1987 & First living related donor kidney transplantation in Hamad General Hospital \\
\hline 1997 & $\begin{array}{c}\text { Qatar national law on Regulating the Human Organs Transfer and } \\
\text { Transplantation }\end{array}$ \\
\hline 1997 & Establishment of CAPD program in Hamad General Hospital \\
\hline 1999 & Frist deceased donor renal transplantation in Hamad General Hospital \\
\hline 2009 & Establishment of nurse assisted home PD program \\
\hline 2010 & $\begin{array}{l}\text { Opening of Fahad Bin Jassim dialysis unit (the largest dialysis unit in the } \\
\text { country) }\end{array}$ \\
\hline 2011 & Inauguration of Qatar Center for Organ Transplantation \\
\hline 2011 & Establishment of structured deceased donor transplantation program \\
\hline 2012 & Opening of the Qatar Center for Organ Donation \\
\hline 2015 & Start of Home HD Dialysis program \\
\hline 2015 & $\begin{array}{c}\text { Revised Qatar national law on Regulating the Human Organs Transfer and } \\
\text { Transplantation }\end{array}$ \\
\hline 2016 & $\begin{array}{l}\text { The nephrology fellowship program accredited by the Accreditation Council } \\
\text { for Graduate Medical Education-International }\end{array}$ \\
\hline
\end{tabular}

Data Source: Qatar's annual renal data 2017.

Nurse-assisted home PD and home HD programs were started in 2009 and 2015 respectively; these programs have helped enhance patients' quality of life and reduce financial burden on the healthcare system.

The first living and deceased donor renal transplants were performed at $\mathrm{HGH}$ in 1987 and 1999 respectively. However, due to the lack of a structured transplant program and scarcity of organ donors, many transplant candidates resorted to commercial kidney transplantation in emerging countries. The Qatar National Law that addresses declaration of brain death and defines penalties for commercialism in organ transplantation was passed in 1997 and revised in 2015. In 2009, Doha Donation Accord was introduced which provided the ethical guidance for deceased and living kidney donor transplantation programs. The Qatar Centre for Organ Transplantation and Qatar Centre for Organ Donation were established in 2011 and 2012 respectively in order to develop national policies for organ donation and transplantation and to oversee all aspects of the program. The nephrology fellowship training program was started in 2010 to augment the supply of a competent healthcare workforce for delivering quality and efficient healthcare services to the expanding ESRD population. The pro- 
gram was accredited by the Accreditation Council for Graduate Medical Education (ACGME) International in 2016.

\section{Epidemiological Transition of RRT-Treated ESRD in Qatar}

Dietary behaviors and sedentary lifestyle patterns that have accompanied the economic development of the country in the last few decades have contributed to increasing rates of obesity in the Qatari community. Obesity appears to be a particular problem for the region of Doha, where almost $40 \%$ of the population is considered obese [3]. This has been coupled with increasing prevalence of chronic non-communicable diseases (NCDs), such as diabetes mellitus and hypertension [4]. Qatar is one of the top ten countries listed as having the highest DM prevalence worldwide [5]. The WHO STEPS survey of 2496 adults conducted in Qatar in 2012 illustrated that an estimated 17\% of the Qatari citizens had diabetes mellitus and 33\% had hypertension [4]. The situation is compounded by the influx of south-Asian immigrants who also tend to have a high prevalence of diabetes and metabolic syndrome [6].

Increasing prevalence of obesity, diabetes mellitus and hypertension-the established risk factors for CKD-has not only increased the burden of CKD and ESRD [4], but has also influenced the statistics on etiology of ESRD in Qatar. The percentages of patients starting RRT for ESRD due to diabetes has increased from 19\% in 1991 to $48 \%$ in 2016 (50 pmp); a trend seen in many gulf countries [7]. Inversely, ESRD due to primary glomerulonephritis has decreased from $33.3 \%$ in 1991 to $13 \%$ (13.6 pmp). In comparison, the unadjusted incident rates of RRT for ESRD due to diabetes and glomerulonephritis have been reported to be 30\% (40 pmp) and 10\% (13 pmp) respectively in Europe [8].

Analysis of Qatar's annual renal data revealed that the prevalence of RRT treated ESRD was 659 pmp on 31st December 2016; 261 new cases of ESRD started RRT in 2016, generating an (unadjusted) incidence rate of $104 \mathrm{pmp}$. This represents a $34 \%$ increase in the incidence of RRT compared to 2013. Although these incidence rates are seemingly lower than those reported in the USA [9], most European countries [8], Australia or New Zealand [10], Qatar's population is significantly distorted demographically, thereby skewing the crude incidence rates. This is due to the fact that the non-citizen community, which forms the major bulk of the total population, is relatively younger; approximately $90 \%$ are in the working-age groups ( 15 to 64 years). Thus, the median age in Qatar is 32.6 years [11]; vs 37.8 years in the USA [12]. Importantly, the segment of population with high ESRD incidence-representing people aged 65 and over-constitutes only $1 \%$ of the total population in Qatar [11] vs $14.5 \%$ in the USA [12].

On December 31, 2016, 44.4\% of all prevalent patients on RRT were receiving HD therapy, $10.7 \%$ were being treated with $\mathrm{PD}$, and $44.4 \%$ had a functioning kidney transplant (Figure 1). Prevalence of these RRT modalities is quite similar to aggregated estimates from Europe [8] and Australia [10]. The United States Renal Data System (USRDS) and Saudi Center for Organ Transplantation 
Prevalent patients on RRT (2016)

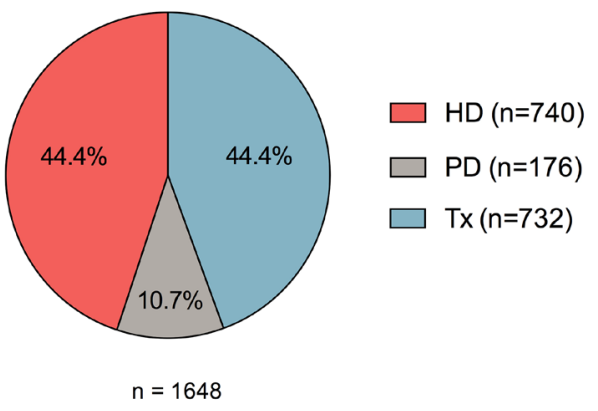

Figure 1. Prevalent patients on RRT on 31st December 2016. HD; hemodialysis, PD; peritoneal dialysis, $\mathrm{Tx}$; functioning renal transplants

(SCOT), in contrast, report that majority of the prevalent patients on RRT receive $\mathrm{HD}(>60 \%)$ and a relatively small $(6 \%-7 \%)$ number of patients utilize PD [9] [13].

Although increasing prevalence of risk factors for CKD are relevant, the dynamics of the non-citizen population as well as socio-economic and RRT organizational factors exert a major influence on the incidence of RRT in Qatar. Similar to other gulf countries, Qatar's non-citizen population is "transient" with a high turnover because of the contractual nature of their employment. This phenomenon skews the medical statistics as many expatriates go back to their countries of origin if a serious illness like ESRD is diagnosed. Conversely, some patients opt to stay in Qatar as they cannot afford RRT in their home countries.

\section{Provision of RRTs in Qatar}

RRT in Qatar is provided only in Ministry of Public Health (MoPH) government hospitals; non-MoPH government or private for-profit $\mathrm{HD}$ facilities do not exist. The government is focusing on health reform by the development of medical insurance but at present, funding is primarily based on HMC healthcare budget. Government health spending per capita is US\$ 2882; the highest in the Middle East \& Africa [9]. It is noteworthy that patients in Qatar have one of the world's lowest burdens of health expenditure, similar to the top performing OECD countries [14]. This is driven by free/highly subsidized public healthcare to all Qatari citizens and non-citizens-a key indicator of equity in removing immediate financial barriers to healthcare.

Dialysis and transplantation services are extended to all citizens and non-citizens (Figure 2). The choice of dialysis takes into account patients' and treatment providers' preferences as well as institutional policies. Companies/individual employers pay the cost of HD for their sponsored workers although some patients are sponsored by charity organizations. A higher percentage of non-citizens is on PD as it is provided free of charge, and is more conducive 


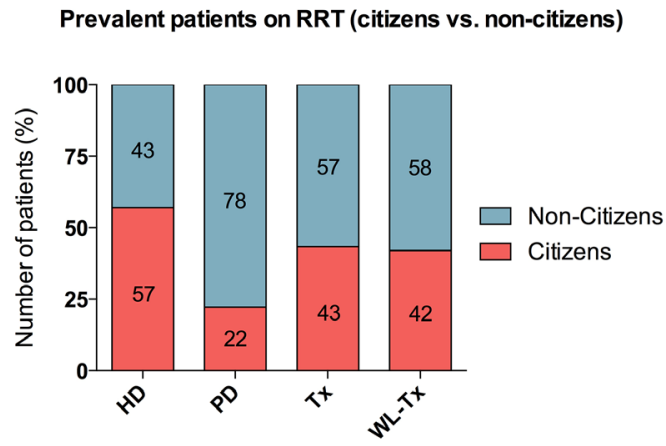

Figure 2. Prevalent patients on RRT on 31st December 2016 (Citizens vs non-citizens). HD; hemodialysis, PD; peritoneal dialysis, Tx; functioning renal transplants, WL-Tx; waitlisted for renal transplantation

to their jobs. Kidney transplantation and follow up services are offered free of charge to all residents in Qatar.

\section{Current Status of RRTs in Qatar}

All major modalities of RRT are available; the geographical locations of the RRT facilities conform to the population density distribution in the country (Figure 3).

\subsection{Hemodialysis}

35 years after the foundation of the first HD unit, the country has 6 HD units to cater for the $80 \%$ of the prevalent dialysis patients who have opted for in-center HD. The biggest unit is in Doha, conforming to the population density of the capital city. It has 80 dialysis stations operating 3 shifts per day. In total, there are $60 \mathrm{HD}$ stations PMP; representing a three-fold increase in HD capacity in the last 10 years. Nine patients are on nurse-assisted home-HD; treatment is rendered by qualified dialysis nurses at patient's home, under the supervision of a nephrologist. Four patients use the traditional machines and five use the portable HD system designed for home use. Biocompatible high flux dialysis membranes and bicarbonate solutions are used; most units use locally produced dialysate prepared according to the specifications of Association for the Advancement of Medical Instrumentation. Qatar's main source of water is desalinated seawater which satisfies $99 \%$ of its municipal water demand. The feed water undergoes further treatment in the dialysis unit water treatment plants to produce dialysis water that meets the AAMI standards for chemical, microbiologic and endotoxin concentrations. Monthly testing for chemical and microbiologic levels is carried out at Central Food Laboratories, Public Health Department, while endotoxin level monitoring is done at each dialysis unit by accredited staff. Analysis of dialysis water quality data during the last 5 year has revealed $<1$ colony-forming units $/ \mathrm{mL}$ and $<0.05$ endotoxin units $/ \mathrm{mL}$. Written policies and 


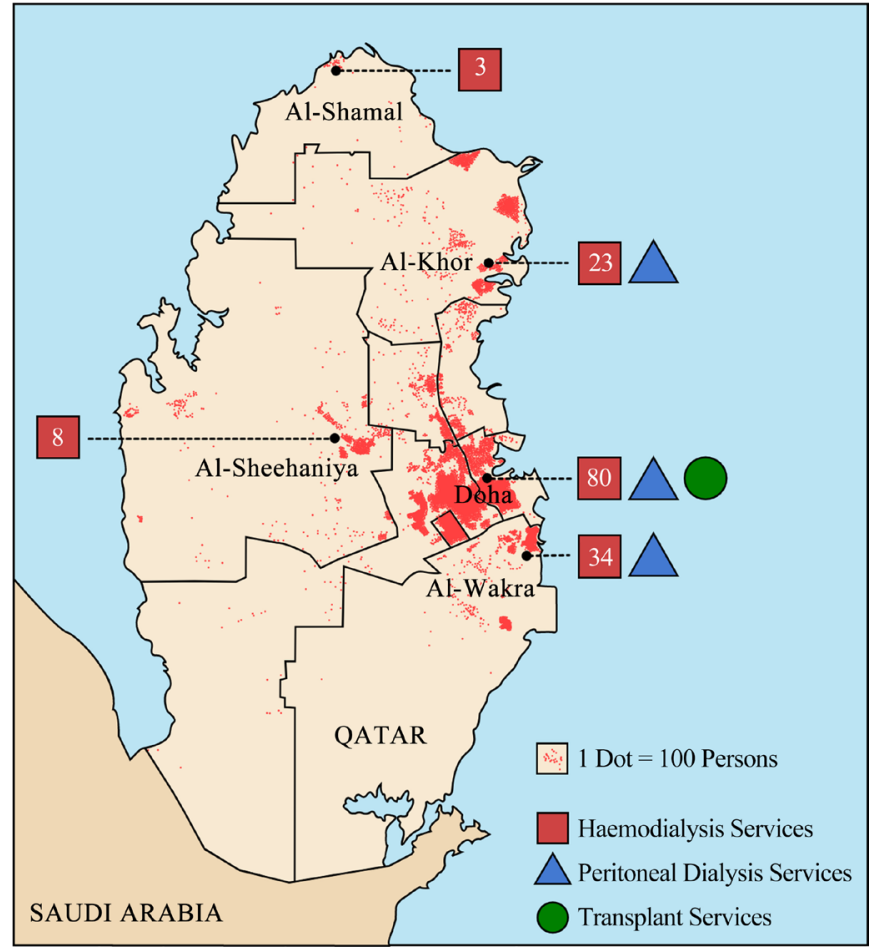

Figure 3. Geographical distribution of RRT facilities in Qatar [2017], in relation to population density [Reference 1]; the values in red squares represent the number of hemodialysis stations at that center [There are two dialysis facilities in Al-Wakra with a total of 34 stations].

procedures are in place covering methodology and responses to results showing unacceptable levels of contaminants.

Qatar participates in the Gulf Cooperation Council-Dialysis Outcomes and Practice Patterns Study (GCC-DOPPS); the recently published results of the phase 5 (2012-2015) study reveal that $92 \%$ of the in-center HD patients dialyze three times per week with mean treatment time of 230 minutes, yielding an average single pool $\mathrm{Kt} / \mathrm{V}$ of 1.5. This schedule is similar to the one practiced in European countries [15], and recommended by European best practice guidelines [16]. Erythropoietin-Stimulating Agents (ESAs) and IV iron prescription is rather liberal (97\% and 73\% respectively), resulting in mean hemoglobin level of $11.1 \mathrm{~g} / \mathrm{dL}$, ferritin $397 \mu \mathrm{g} / \mathrm{L}$ [397 ng/ml] and transferrin saturation 30.7\% [17]. Similarly, judicious use of phosphate binders and Cinacalcet has facilitated achievement of the NKF/K-DOQI recommended target values for bone and mineral metabolism in majority of the patients. The mean serum calcium is 2.2 $\mathrm{mmol} / \mathrm{L}$ [8.9 mg/dL], serum phosphorus $1.5 \mathrm{mmol} / \mathrm{L}$ [ $4.5 \mathrm{mg} / \mathrm{dL}$ ] and PTH, 370 $\mathrm{ng} / \mathrm{L}[370 \mathrm{pg} / \mathrm{mL}]$ [18]. Availability of expensive medication such as ESAs, Sevelamer and Cinacalcet at subsidized price has contributed to their widespread use.

The unadjusted 1-year and 5-year survival rates of the HD patients have previously been reported to be $84 \%$ and $53 \%$ respectively[19], which is comparable with or superior to larger international registries (USRDS, $77.7 \%$ and $42.6 \%$ ) [8] 
[9].

\subsection{Peritoneal Dialysis}

Since its initiation in 1997, PD has been increasingly utilized as a mode of RRT. Currently (July 2017), 197 patients (10.7\% of RRT patients; $20 \%$ of the prevalent dialysis patients) are maintained on PD; the major bulk of which are followed up in Doha although smaller programs also operate in $\mathrm{Al} \mathrm{Wakra} \mathrm{and} \mathrm{Al}$ Khor cities. The percentage of PD utilization in Qatar is significantly higher than that in the USA (6.9\%), Europe (5\%) or KSA (6\%) [8] [9] [13]. This is likely due to cost effectiveness of PD, availability of nurse assisted-home PD as well as in-depth training of the young nephrologists in PD which influences their prescription habits with regards to dialysis modality.

Use of APD has grown markedly in the recent years due to the lifestyle, solute clearance and fluid removal advantages it offers, particularly in fast transporters $-50 \%$ of the PD patients utilize this modality, which is comparable to the trend in most European countries and New Zealand [8] [10]. In contrast, APD usage is substantially higher in Australia (66\%), and the Kingdom of Saudi Arabia (KSA) (70\%) [10] [13].

The introduction of nurse-assisted home PD in 2009 represented a key milestone-Qatar was the first country in the Middle East to introduce such a program. It offers an attractive home-based dialysis option to the elderly patients who have physical, cognitive or psychological barriers to self-care PD. All patients in this program are treated with APD; a trained nurse helps with the setting up of the cycler, connecting and disconnecting patients from the cycler at their homes. This program has increased the utilization of PD and reduced the need for in-center HD.

Overall unadjusted 1-year and 2-year survival (from day 1) is $91 \%$ and $77 \%$ respectively [20] which compares favorably to European Dialysis and Transplant Association (EDTA) registry data [8]. Exclusive use of the twin-bag system and a strong focus on patient training and quality improvement, has kept the peritonitis rate low at 0.2 episodes per patient-year.

\subsection{Kidney Transplantation}

Structured, Joint Commission International accredited deceased donor and living kidney donor transplant programs operate at Hamad General Hospital, backed up by a multidisciplinary team of credentialed transplant surgeons and nephrologists, transplant coordinators, modern operating theatre and ICU facilities, as well as radiology, histopathology and HLA laboratory services. Laparoscopic living donor nephrectomy was introduced in 2013 which has reduced the donor morbidity and hospital stay.

Legal and policy framework of Qatar's transplant program intensely prohibits organ commercialism. Violation of the Qatari national law on Regulating the Human Organs Transfer and Transplantation is punishable by monetary penal- 
ties, imprisonment, temporary suspension of license to practice or closure of the facility involved. On the other hand, the program promotes safe and ethical living kidney transplantation [21]. Equal transplant opportunities are provided to citizens and noncitizens, regardless of their social/ethnic background; all transplant candidates are placed on a unified waiting list (Figure 2). Policies and clinical guidelines are in place to ensure consistency in donor and recipient selection criteria, evaluation process, organ allocation and surgical care. Financial disincentives for ethical organ donation and transplantation have been minimized so that perioperative care, long-term medical follow-up and immunosuppressive medication are provided free of charge to all residents in Qatar.

In the past, many transplant candidates from Qatar had been motivated to seek commercial transplantation abroad. Implementation of legislation against organ trade and collaboration with international healthcare institutions for overseas live related transplantation of Qatari citizens has reduced commercial kidney transplantation abroad by $65 \%$ [22]. It has also led to a change in attitudes of public towards organ donation such that more people are willing to consider live related kidney transplantation. Shifting the public's mindset towards deceased donation has proved difficult although the national donor registry, launched in 2012, reached 178, 450 registrants in April 2017 (approximately $10 \%$ of the eligible adult population).

Kidney transplant rates increased from $2.5 \mathrm{pmp}$ in 2008 to $21 \mathrm{pmp}$ in 2016. Although these rates are significantly lower than those in Europe (36 pmp), [8] by equipping the country with the legislation necessary to establish deceased donor transplantation program and removing the obstacles to living kidney donation, Qatar is progressing towards achieving national self-sufficiency in donor kidneys for transplantation. On 31st December 2016, 44.4\% of all prevalent ESRD cases in Qatar had a functioning kidney transplant (Figure 1). Currently, there are 721 kidney transplant recipients (313 citizens and 408 non-citizens) who receive regular medical follow-up at HGH.

\section{Nephrology Workforce}

There is evidence that care by nephrologists generally leads to better patient and renal outcomes [9] [23]. Unfortunately, international data suggests that trained nephrology workforce has not grown at pace with the growing disease burden and healthcare needs of patients with kidney disease [24]. In the past Qatar had relied heavily on global recruitment of foreign-trained physicians to tackle staff shortages. Careful nephrology workforce planning led to the commencement of a nephrology fellowship program in Qatar in 2010. This program, which has received accreditation from ACGME International, is proving to be a continuing source of nephrologists proficient in the management of renal disease in ambulatory, in-patient and intensive care settings. The number of nephrology consultants has increased from 1.5 pmp in 2000 to 7 pmp in 2016 [ 1 consultant nephrologist for each 100 RRT-treated ESRD patients]. 


\section{Education and Quality Improvement}

Given that education of physicians and patients reduces the risk of progression of kidney disease and/or death [25], HMC has devoted substantial resources towards medical education and research. Qatar regularly holds international nephrology conferences, symposia, and workshops which provide continuing medical education opportunities to not only nephrologists but also general physicians, endocrinologists and cardiologists, who are more likely to encounter patients in earlier stages of CKD. These events are also an important source of broader dissemination of international best practice guidelines [such as those produced by the National Kidney Foundation (NKF), Kidney Disease Outcomes Quality Initiative (K/DOQI) or Kidney Disease Improving Global Outcomes (KDIGO)]. In addition, a variety of public events are organized, particularly on World Kidney Day to raise awareness of risk factors of CKD and preventive behaviors. The Nephrology division has been successful in obtaining grant funding from Qatar National Research Fund for basic and clinical research in diabetic kidney disease.

Recently, there has been an increasing emphasis on healthcare quality initiatives that are based on key performance indicators (KPIs). Each year, a clinically relevant set of KPIs is implemented, enabling each hospital/dialysis unit to determine its performance against consensus clinical practice guidelines. KPIs that have been monitored include use of statins in CKD patients, timely creation of vascular access for $\mathrm{HD}$, correction of anemia, control of serum calcium/phosphorus/hyperparathyroidism, incidence of PD peritonitis, and surveillance of post-transplant diabetes mellitus \& malignancy. It is felt that tracking of KPIs impacts positively on care and outcomes of patients with CKD.

\section{From Treatment to Prevention of CKD and ESRD—Paradigm Shift in Health Care}

To reduce the incidence of ESRD, it is crucial to detect CKD early in its course, as it will provide opportunities for treatment of reversible factors, better management of comorbidities and slowing the rate of progression to ESRD. The $\mathrm{MoPH}$ aims to improve awareness and services for diabetes and obesity [14] and has formulated national guidelines for increasing physical activity. A variety of awareness programs such as "We Are Healthy Kids" and "Workplace Wellness Program" have been introduced. In addition, a national diabetes strategy and action plan has been launched.

As part of the national primary health care strategy 2013-2018, the Primary Health Care Corporation (PHCC) is implementing comprehensive screening programs for diabetes, hypertension and kidney disease. This is important because PHCC provides 39\% of Qatar's outpatient care. The MoPH has also produced evidence-based algorithms/pathways on screening, diagnosis and management of CKD for primary healthcare providers. These pathways also provide guidance to the primary care physicians regarding timing of referral of patients 
for evaluation/management to the nephrologists. It is believed that early diagnosis and management of the NCDs will reduce the burden of kidney disease in Qatar and provide cost savings for the health care system.

\section{Future of RRT in Qatar; A Glance into the Next Decade}

In the future, prevention of CKD and ESRD will become one of the priority areas of public health. The campaign against obesity, diabetes and hypertension will build up substantially and bring together leaders from academia, industry and policy makers of public health. Interventions targeting hypertension, diabetes mellitus and proteinuria with a standardized pharmacological therapy will be implemented rigorously in primary care and supported by specialized hospital care.

Preventative strategies for CKD will take several years to make any meaningful impact-hence it is predictable that the prevalence of RRT-treated ESRD population will increase with time. Current trends in utility of RRT modalities suggest that HD will remain the dominant modality for dialysis in future, necessitating expansion of existing facilities and establishment of new ones. In view of growing cost constraints, it is conceivable that the use of home based therapies-HD and PD will increase appreciably. Patients will be trained comprehensively to perform hemodialysis independently at home, without the assistance of a dialysis nurse. The Qatari government is encouraging the private sector to play a greater role in healthcare provision; it is likely that RRT services will also be offered at private healthcare facilities, especially with the introduction of health insurance. A unified national renal registry database will be established for tracking demographic, clinical and laboratory data, estimating disease burden and monitoring the quality of care. This registry database will also be used for future research and strategic planning for RRT services in Qatar.

Renal transplantation, in particular pre-emptive renal transplantation will increase considerably. The transplant program has developed policies and protocols for desensitization and $\mathrm{ABO}$ incompatible transplantation; this will benefit approximately one third of the living donor-recipient pairs in the program who are immunologically incompatible with each other. More renal transplants will be carried out locally as the national and international policies on commercial transplantation become more stringent. Availability of deceased kidney donors will remain a key challenge. Current estimates reveal that family consent rates for organ donation from deceased donors remain at less than 20\% [22]. Public awareness campaigns and training of ICU physicians and transplant coordinators will hopefully expand the pool of deceased donors. Expanded criteria deceased organ donors will be utilized more liberally, hence more patients will benefit from transplantation. Donation after cardiac death and kidney paired donation programs will start to maximize transplant rates.

The number of nephrologists will increase to at least $10 \mathrm{pmp}$ or 1 per 75 RRT-treated ESRD patients, approaching the scale among European countries 
and the USA.

\section{Conclusions}

Qatar has experienced rapid evolution of RRT in the last four decades. All major modalities of RRT are available, all providing therapeutically effective and individualized care to patients with ESRD, at par with international standards. The services are accessible to all communities independent of their ethnicity or socioeconomic status.

Given the significant rise in the prevalence of NCDs and RRT, it is predictable that $\mathrm{HD}$ and CAPD population will continue to grow, requiring development of new dialysis units. However, the government and nephrology community realize that a multipronged integrated approach is required to combat ESRD. It is vital to continue to strengthen policies and programs on healthy lifestyles and NCD prevention, and intensify the efforts to aggressively treat CKD in its early stages at community level. At the same time, it is imperative that further progress in kidney transplantation is made by taking on board new initiatives to expand the organ donor pool and optimizing the use of available donors. Finally, it is incumbent that the nephrology workforce is reinforced. Effective education, training, and professional development of nephrologists, transplant coordinators, dialysis nurses, and technicians will make the greatest difference in providing safe, effective, and compassionate care to every patient with renal disease-reflecting the mission of Hamad Medical Corporation.

\section{Acknowledgements}

We thank Nephrology and Dialysis staff of HMC for providing relevant data, and Hania Asim for her assistance with computer graphics.

\section{Grants/Funding}

This research did not receive any specific grant from funding agencies in the public, commercial, or not-for-profit sectors.

\section{References}

[1] Qatar Population and Social Statistics. http://www.mdps.gov.qa

[2] Global Finance Magazine. https://www.gfmag.com/global-data

[3] Ali, F.M.H., Nikoloski, Z., Reka, H., Gjebrea, O. and Mossialos, E. (2014) The Diabetes-Obesity-Hypertension Nexus in Qatar: Evidence from the World Health Survey. Population Health Metrics, 12, 18. https://doi.org/10.1186/1478-7954-12-18

[4] WHO STEPS Chronic Disease Risk Factor Surveillance (2012) Qatar STEPS Survey Report. http://www.who.int/chp/steps/qatar

[5] International Diabetes Federation (2013) International Diabetes Federation Diabetes Atlas. http://www.idf.org/diabetesatlas/

[6] Ramachandran, A., Snehalatha, C., Shetty, A.S. and Nanditha, A. (2012) Trends in Prevalence of Diabetes in Asian Countries. World Journal of Diabetes, 3, 110-117. https://doi.org/10.4239/wjd.v3.i6.110 
[7] Al Sahow, A., AlRukhaimi, M., Al Wakeel, J., et al. (2016) GCC-DOPPS 5 Study Group. Demographics and Key Clinical Characteristics of Hemodialysis Patients from the Gulf Cooperation Council Countries Enrolled in the Dialysis Outcomes and Practice Patterns Study Phase 5 (2012-2015). Saudi Journal of Kidney Diseases and Transplantation, 27, S12-23. https://doi.org/10.4103/1319-2442.194885

[8] Annual Report (2014) ERA-EDTA Registry. https://www.era-edta-reg.org

[9] 2015 USRDS Annual Data Report. Volume 2: End-Stage Renal Disease. https://www.usrds.org

[10] The 39th Annual ANZDATA Australia and New Zealand Dialysis and Transplant Registry Report (2016). http://www.anzdata.org.au

[11] Qatar Population. http://countrymeters.info

[12] United States Census Bureau. https://www.census.gov

[13] SCOT Data (2015) Dialysis in the Kingdom of Saudi Arabia. Saudi Journal of Kidney Diseases and Transplantation, 23, 839-848

[14] SCH Annual Report 2014. https://www.moph.gov.qa

[15] Couchoud, C., Kooman, J., Finne, P., et al. (2009) Quality European Studies Working Group on Dialysis Adequacy. From Registry Data Collection to International Comparisons: Examples of Haemodialysis Duration and Frequency. Nephrology Dialysis Transplantation, 24, 217-224. https://doi.org/10.1093/ndt/gfn442

[16] Tattersall, J., Martin-Malo, A., Pedrini, L., et al. (2007) EBPG Guideline on Dialysis Strategies. Nephrology Dialysis Transplantation, 22, ii5-ii21.

https://doi.org/10.1093/ndt/gfm022

[17] Abouchacra, S., Obaidli, A., Al-Ghamdi, S.M., et al. (2016) Gulf Cooperation Council-Dialysis Outcomes and Practice Patterns Study: An Overview of Anemia Management Trends at the Regional and Country Specific Levels in the Gulf Cooperation Council Countries. Saudi Journal of Kidney Diseases and Transplantation, 27, 51-61. https://doi.org/10.4103/1319-2442.194895

[18] Al Salmi, I., AlRukhaimi, M., AlSahow, A., et al. (2016) Mineral Bone Disorder and Its Management among Hemodialysis Patients in the Gulf Cooperation Council: Initial Findings from the Dialysis Outcomes and Practice Patterns Study (2012-2015). Saudi Journal of Kidney Diseases and Transplantation, 27, 62-80. https://doi.org/10.4103/1319-2442.194902

[19] Shigidi, M.M., Ramachandiran, G., Rashed, A.H. and Fituri, O.M. (2009) Demographic Data and Hemodialysis Population Dynamics in Qatar: A Five Year Survey. Saudi Journal of Kidney Diseases and Transplantation, 20, 493-500.

[20] Shigidi, M.M., Fituri, O.M., Chandy, S.K., Asim, M., Al Malki, H.A. and Rashed, A.H. (2011) Peritoneal Dialysis, an Expanding Mode of Renal Replacement Therapy in Qatar. Saudi Journal of Kidney Diseases and Transplantation, 22, 587-593.

[21] Asim, M., Al-Maslamani, Y. and Al-Malki, H. (2017) Safe and Ethical Living Kidney Donation in Qatar: A National Health System's Approach. Qatar Medical Journal, 2017, 3. https://doi.org/10.5339/qmj.2017.3

[22] Almaslamani, Y., Almalki, H., Fadhil, R., Khalaf, H. and Asim, M. (2016) Transplantation in Qatar. Transplantation, 100, 2487-2488. https://doi.org/10.1097/TP.0000000000001475

[23] Osinski, M.H. and Kirschner, M.J. (1997) Is There a Nephrologist in the House? Nephrology News \& Issues, 15-8, 20.

[24] Sharif, M.U., Elsayed, M.E. and Stack, A.G. (2016) The Global Nephrology Work- 
force: Emerging Threats and Potential Solutions! Clinical Kidney Journal, 9, 11-22. https://doi.org/10.1093/ckj/sfv111

[25] Cortés-Sanabria, L., Cabrera-Pivaral, C.E., Cueto-Manzano, A.M., et al. (2008) Improving Care of Patients with Diabetes and CKD: A Pilot Study for a Cluster-Randomized Trial. American Journal of Kidney Diseases, 51, 777-788.

https://doi.org/10.1053/j.ajkd.2007.12.039 